\title{
Small fermionic systems: the common methods and challenges
}

\author{
J. Navarro*, P.-G. Reinhard ${ }^{\dagger}$ and E. Suraud** \\ * IFIC (CSIC-Universitat Valencia) Apdo. 22085 E-46071 Valencia, Spain email \\ ${ }^{\dagger}$ Institut für Theoretische Physik, Universität Erlangen, Staudstrasse 7, D91058 Erlangen, Germany \\ ${ }^{* *}$ Laboratoire Physique Théorique, Université Paul Sabatier, 118 Route de Narbonne, 31062 Toulouse cedex,
}

France

\begin{abstract}
We discuss three finite Fermion systems in comparison: nuclei, metal clusters, and droplets of liquid ${ }^{3} \mathrm{He}$. A principle sorting in "natural units" of energy and length scales is given. We address the theoretical description in terms of self-consistent mean-field theories and their effective energy-density functionals. We look at the interplay of the different time scales from the various constituents of either system. Finally, we discuss the prospects of more detailed experimental analysis for the case of metal clusters, in particular in the non linear domain where truly dynamical behaviors are expected.
\end{abstract}

Keywords: Nuclei, Metal Clusters, liquid He droplets

PACS: $25.70 .-\mathrm{z}, 36.40 .-\mathrm{c}, 67.55 .-\mathrm{s}$

\section{INTRODUCTION}

Finite Fermion systems are droplets of Fermi liquid. Typical examples are nuclei, ${ }^{3} \mathrm{He}$ droplets, and metal clusters. Fermi liquids constitute one of the basic states of matter [1]. They are highly correlated systems which, however, never freeze out to a crystalline state. They have a well defined saturation density with low (nuclei and metal clusters) or moderate (liquid ${ }^{3} \mathrm{He}$ ) compressibility. The finite drops thus share several key features: scaling of radius with $N^{1 / 3}$, shell effects (magic numbers, Jahn-Teller deformation, for clusters see [2]), pronounced resonance excitations (giant resonances, plasmons [3]), and fusion/fission [4]. The strong correlations can hardly be dealt with in detail. Effective energy-density functionals are employed for self-consistent calculations of ground state and dynamics, see e.g. [5] for the electrons in clusters, [6] for nuclei, and [7] for ${ }^{3} \mathrm{He}$ droplets. This short list of basic properties shows that finite Fermion systems have much in common. On the other side, there are several noteworthy differences, e.g., concerning composition or relation of time scales. It is thus most interesting to discuss these systems in comparison. It is the aim of this contribution to provide such a discussion in due brevity. Thereby, we will concentrate on the energetic dynamical aspects and refer to [8] for structure and the low-energy domain. We will first compare the three systems concerning typical scales (length, time, energy), construction of effective energy-density functionals, and available data. In a last section, we will discuss briefly observables from non-linear dynamics for the particular example of metal clusters. A much more extensive discussion of practically all aspects of cluster dynamics can be found in [9].

\section{NUCLEI, ${ }^{3}$ HE DROPLETS AND METAL CLUSTERS}

Nuclei, helium droplets and the electron cloud of metal clusters are dense fermion systems with strong Pauli correlations. For a more quantitative discussion, let us briefly recall a few key characteristics of nuclei, metal clusters and helium droplets, concerning, in particular, dominant interactions, sizes, structure and dynamics. These characteristics are briefly sketched in table 1 . In metal clusters, the Coulomb interaction plays an important role. The repulsive interactions between electrons are compensated by the attraction to ions. In a neutral cluster, it is finally the electronic exchange and correlation part of the interaction which provides most of the binding. In nuclei, the binding is dominated by the short range nuclear interaction providing more than enough binding to overrule the repulsive Coulomb interaction which grows with the number of protons. In helium droplets, the interaction, originally coulombic, reduces to a mere (extremely faint) interaction of van der Waals form leading to extremely fragile structures. All three systems furthermore exhibit a "saturating" behavior. Their radii scale with the power $1 / 3$ of the size of the system. The proportionality factor is the Wigner Seitz radius $r_{s}$, often denoted as $r_{0}$ in case of nuclei. This means that each 
TABLE 1. Gross characteristics of nuclei, metal clusters and helium droplets. One successively considers the constituents (all fermions but for the ions in clusters), the interactions at play, the radii of systems of sizes $A$ (nuclei) and $N$ (clusters, helium droplets), the typical distance between constituents, the typical mean free path and de Broglie wavelength as estimated from a Fermi gas picture of the ground state. Distances are expressed in terms of $r_{0}$ for nuclei and $r_{s}$ for clusters and helium droplets. The parameter $r_{0}$ is the parameter entering systematics of nuclear radii; the parameter $r_{s}$ is the Wigner Seitz radius of the material constituting the metal clusters or the helium droplets.

\begin{tabular}{|c|c|c|c|}
\hline & Nuclei & Clusters & Helium \\
\hline Constituents & $\begin{array}{l}N \text { Neutrons } \\
Z \text { Protons }\end{array}$ & $\begin{array}{l}N \text { Electrons } \\
N_{\text {ions }} \text { Ions }\end{array}$ & $\begin{array}{l}N^{3} \mathrm{He} \text { atoms } \\
(2 p, 1 n, 2 e)\end{array}$ \\
\hline Interaction & $\begin{array}{l}\text { SR (nuclear) } \\
+ \text { LR (Coulomb) }\end{array}$ & LR (Coulomb) & SR van der Waals \\
\hline Size & $N+Z=A \leq 300$ & $3 \leq N \leq 10^{5-7}$ & $30 \leq N$ \\
\hline Radius & $\begin{array}{l}R \sim r_{0} A^{1 / 3} \\
r_{s} \equiv r_{0} \sim 1.2 \mathrm{fm}\end{array}$ & $\begin{array}{l}R \sim r_{s} N^{1 / 3} \\
r_{s} \sim 0.1-0.3 \mathrm{~nm}\end{array}$ & $\begin{array}{l}R \simeq r_{s} N^{1 / 3} \\
r_{0} \sim 0.25 \mathrm{~nm}\end{array}$ \\
\hline Distance constituents & \multicolumn{3}{|c|}{$d \sim 1.5-2 r_{0, s}$} \\
\hline Mean free path & \multicolumn{3}{|c|}{$\lambda \sim R$} \\
\hline de Broglie wavelength & \multicolumn{3}{|c|}{$\lambda_{B} \sim \pi r_{0, s}$} \\
\hline Fermi energy $\varepsilon_{F}$ & $40 \mathrm{MeV}$ & $1.4-12 \mathrm{eV}$ & $5 \mathrm{~K}$ \\
\hline
\end{tabular}

fermion occupies the same volume given by $(4 / 3) \pi r_{s}^{3}$. And this also implies that the average density of these systems is $\rho \sim 3 /\left(4 \pi r_{s}^{3}\right)$ independent of the system size. The parameters $r_{0}, r_{s}$ thus play a key role in fixing the characteristic scales in these systems. One can for example estimate the typical distance between constituents, which amounts to about 1.5-2 $r_{s}$. One can also evaluate the mean free path. In all cases it turns out to be of the order of magnitude of the actual size of the system, so that one can adopt the view that the fermions evolve nearly independent from each other, which motivates a mean field approach. Taking for sake of simplicity a Fermi gas picture (which serves reasonably well as a first approximation) one can also introduce an energy scale in terms of $r_{s}$ through the Fermi momentum $k_{F}=\left(3 \pi^{2} \rho\right)^{1 / 3}=\left(9 \pi / 4^{1 / 3} r_{s}^{-1}\right.$ from which one can deduce a typical Fermi energy $\varepsilon_{F}=\left(\hbar^{2} / 2 m\right) k_{F}^{2}$ and Fermi velocity $v_{F}=\hbar k_{F} / m$ (with $m=m_{n}, m_{e}, m_{H e}$ ). This by the way also provides a simple estimate of the de Broglie wavelength in the ground state $\lambda_{B} \sim 2 \pi / k_{F} \sim \pi r_{s}$, which confirms the essentially quantal nature of these systems.

A comparison of mean-fields is presented in figure 1 for the cluster $\mathrm{Na}_{40}$, the nucleus ${ }^{78} \mathrm{Sr}$ (with 40 neutrons) and a helium droplet with $40{ }^{3} \mathrm{He}$ atoms. The results are plotted in natural units (see figure caption) for making the systems comparable. The comparison is quite enlightening. First one notes that all three systems fit into one figure, i.e. have about the same scales when expressed in natural units. Moreover, they exhibit the same spatial extension, directly 


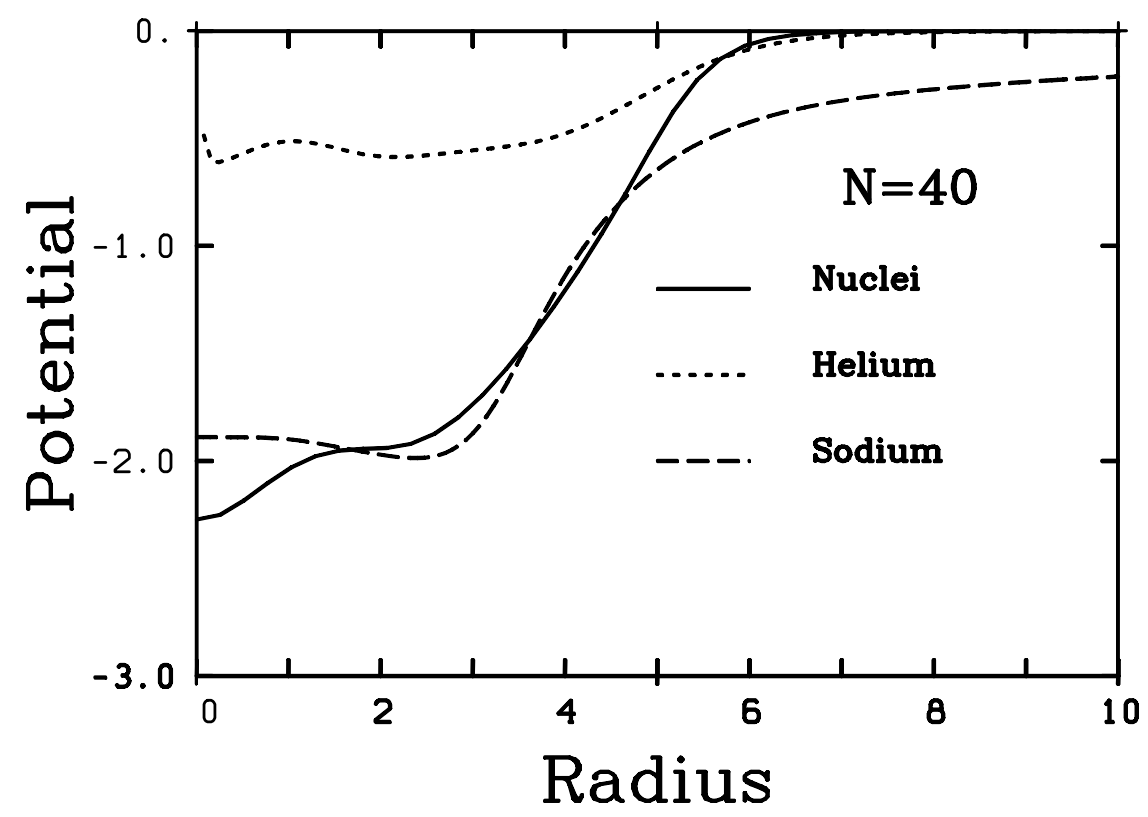

FIGURE 1. The mean-field potentials for a Na cluster, a nucleus and an helium droplet, for 40 particles (the results are only shown for the neutron part in the nuclear case). Natural units are used: lengths in units of $r_{s}$ and energies in units of $\varepsilon_{F}$.

connected to the "saturation scale" introduced by $r_{s}$. At second glance, one also spots differences, in the depth of the potential wells and in the asymptotic behaviors. The cluster and nucleus share a comparably deep potential while the helium droplet exhibits a much more shallow potential well reflecting the faintness of the interaction between two He atoms. On the other hand, the helium droplet and the nucleus share the same asymptotic behavior characteristic of a system dominated by a relatively short range interaction, while the cluster case exhibits a typical long range coulomb behavior. All in all the comparison nevertheless shows the overall similarity between the various systems, up to details.

\section{MEAN-FIELD: A POSSIBLE COMMON THEORY}

The free nucleon-nucleon interaction is known to be strongly repulsive at short range [10]. This makes a mean field theory a priori questionable. But the strong Pauli correlations in nuclei significantly suppress low energy scattering, which renormalizes, in a nuclear medium, the interaction to an effective one. The short distance repulsion is thus highly suppressed and the effective nucleon-nucleon interaction smooth enough to justify a mean field picture [10]. The so called Skyrme interactions, which integrate in an effective way, these Pauli correlation effects on a basically zero range bare interaction, have become since decades a standard microscopic tool in the field, because of their simplicity and because of the many successes they have allowed, at least for stability valley nuclei, for a recent review see [6]. As can be seen from table 2 the Skyrme interaction appears as a density functional, mostly local, non locality being usually assumed in terms of a gradient expansion.

A somewhat similar reasoning applies to metal clusters. The general atomic problem is indeed singular (due to the point charge of the atomic nucleus), but a limited number of valence electrons actually take part in the binding of molecular systems or clusters. This is especially true in the case of simple metals as the valence shell is well separated from core levels and usually little bound. Valence electrons can thus easily be delocalized to form the rather "soft" metal bonds. This, by the way, also allows to "pack" core electrons into a pseudo potential: this reduces the many-electron problem to the treatment of the valence electrons only in a reasonably smooth ionic background [9]. This again provides a favorable situation for a mean field treatment. The success of the many calculations based on Density Functional Theory (DFT) even in its simplest Local Density Approximation (LDA) version indeed proves the reliability of the mean field approach.

The case of helium droplets is a bit more involved. Indeed the applicability of a DFT approach was long debated for such systems in view of the much stronger correlations. However, it seems today that density functionals can indeed be 
TABLE 2. One-body Hamiltonian used in mean field calculations in nuclei (Skyrme like interaction), metal clusters (DFT LDA) and helium droplets (DFT). In nuclei the density $\rho$ represents the neutron and/or proton density, the latter entering the coulomb interaction alone $\left(\rho_{p}\right)$. In clusters the density is the electronic one while in helium it is the density of helium atoms. In the case of cluster electrons an important contribution comes from the exchange correlation potential $U_{\mathrm{xc}}[\rho]$ which can be treated in the simplest LDA approximation. The external potential $U_{\text {ext }}(\mathbf{r}, t)$ typically refers to the fields as generated by ions or a possible external field (laser, by passing ion...). In the case of helium droplets one has to introduce an effective mass $m^{*}$ as indicated, which again depends on the density. The same is true in principle for the nuclear case but the effective mass is much closer to the bare mass (about $20 \%$ ) than in the helium case (factor 3 typically).

\begin{tabular}{|l|l|}
\hline Nuclei & $h[\rho]=-\nabla \frac{\hbar}{m^{*}(r)} \nabla+t_{0} \rho+t_{3} \rho^{1+\sigma}+t_{12}(\nabla \rho)^{2}+\int \frac{\rho_{p}\left(\mathbf{r}^{\prime}\right)}{\left|\mathbf{r}-\mathbf{r}^{\prime}\right|} d \mathbf{r}^{\prime}+\ldots$ \\
\hline Clusters & $h[\rho]=-\frac{\hbar^{2}}{2 m} \Delta+\int \frac{\rho\left(\mathbf{r}^{\prime}\right)}{\left|\mathbf{r}-\mathbf{r}^{\prime}\right|} d \mathbf{r}^{\prime}+U_{\mathrm{xc}}[\rho]+U_{\text {ext }}(\mathbf{r}, t)$ \\
\hline Helium & $\begin{array}{l}h[\rho]=-\nabla \frac{\hbar^{2}}{m^{*}(r)} \nabla+\int V_{e f f}\left(\mathbf{r}-\mathbf{r}^{\prime}\right) \rho\left(\mathbf{r}^{\prime}\right) d \mathbf{r}^{\prime}+A \rho^{2+\gamma}+\ldots \\
\frac{\hbar^{2}}{2 m^{*}}=\frac{\hbar^{2}}{2 m}+\alpha \rho+\beta \rho^{2}\end{array}$ \\
\hline
\end{tabular}

used in that case provided one introduces a finite range in the construction $[7,11]$. Once taking that step, the situation is even more favorable than in case of nuclei. The ab initio calculations for ${ }^{3} \mathrm{He}$ matter have converged to generally accepted, satisfying degree of reliability [12] and there exists experimental access to bulk ${ }^{3} \mathrm{He}$ over a wide range of pressures [13]. Both facts provide well tested data as input for a proper calibration of density functionals. There exist even ab initio calculations for finite droplets which serve as additional benchmark [14].

In the majority of practical cases the mean-field calculations based on the one-body hamiltonians presented in table 2 are done at quantum level. Each particle (nucleon, electron, helium atom) is attributed a one particle wavefunction $\phi_{i}(\mathbf{r})$, from which one deduces the single particle density matrix $\hat{\rho}\left(\mathbf{r}, \mathbf{r}^{\prime}\right)$ and the local one-body density $\rho(\mathbf{r})=$ $\hat{\rho}(\mathbf{r}, \mathbf{r})=\sum_{i}\left|\phi_{i}(\mathbf{r})\right|^{2}$ (where the summation runs over all particles). The one-body wavefunctions then follow an effective Schrödinger equation

$$
i \hbar \frac{\partial \mid \varphi_{i}>}{\partial t}=h[\rho(\mathbf{r})] \mid \varphi_{i}>
$$

with an effective single-particle Hamiltonian $h$ expressed as a functional of the density $\rho(\mathbf{r})$, as given in table 2 for the various Fermi systems. This mean field equation can be recast in the equivalent matrix form

$$
i \hbar \hat{\rho}=[h, \hat{\rho}] \quad .
$$

There also exist semi-classical approximations to this quantum scheme. It can be "formally" obtained by transforming the density operator $\hat{\rho}$ into a one-body phase space distribution $f(\mathbf{r}, \mathbf{p}, t)$, which becomes the basic ingredient, and the commutator into Poisson brackets:

$$
\begin{array}{ccc}
\hat{\rho}\left(\mathbf{r}, \mathbf{r}^{\prime}\right) & \longrightarrow & f(\mathbf{r}, \mathbf{p}, t) \\
{[., .]} & \longrightarrow & \{., .\}
\end{array}
$$

This leads to the Vlasov equation

$$
\frac{\partial f}{\partial t}=\{h, f\}
$$

The one-body Hamiltonian has the same expression in terms of the density $\rho(\mathbf{r})$ as in the quantal form, but the density is now computed from the phase space density as

$$
\rho(\mathbf{r}, t)=\int d^{3} p f(\mathbf{r}, \mathbf{p}, t)
$$


TABLE 3. Schematic status of studies for the three finite Fermion systems. The entry "no $\rightarrow$ yes" indicates that research is underway and results may show up soon. The entry "not yet" means that calculations have not been done but are feasible in principle. See text for details.

\begin{tabular}{|l|c|c|c|c|}
\hline \multirow{2}{*}{ system } & \multicolumn{2}{|c|}{ experiment } & \multicolumn{2}{|c|}{ theory } \\
& structure & dynamics & structure & dynamics \\
\hline nuclei & yes & yes & yes & yes \\
\hline clusters & yes & no $\rightarrow$ yes & yes & no $\rightarrow$ yes \\
\hline 3ैHe droplets & no $\rightarrow$ yes & no & yes & not yet \\
\hline
\end{tabular}

This equation can then be extended to account for dynamical correlations Particle-particle scattering effects can indeed easily be included as a Markovian collision term for the phase space distribution $f$. This has been worked out in great detail in nuclear physics applications [15] and it was also extended to the cluster case. In both cases (nuclei, metal clusters) one ends up with the VUU (Vlasov Uehling Ulhenbeck) equation

$$
\frac{\partial f}{\partial t}+\frac{\mathbf{p}}{m} \frac{\partial f}{\partial \mathbf{r}}-\frac{\partial V}{\partial \mathbf{r}} \frac{\partial f}{\partial \mathbf{p}}=I_{U U}(\mathbf{r}, \mathbf{p}, t)
$$

with the collision term

$$
\left.\left.I_{U U}=\int \frac{d^{3} p_{2} d \Omega}{(2 \pi \hbar)^{3}} \frac{d \sigma}{d \Omega}\left|v_{12}\right|\left\{f_{1} f_{2}\left(1-f_{3} / 2\right)\left(1-f_{4} / 2\right)\right)-f_{3} f_{4}\left(1-f_{1} / 2\right)\left(1-f_{2} / 2\right)\right)\right\}
$$

where $v_{12}$ is the relative velocity of the colliding particles 1 and 2 . The differential cross section $d \sigma / d \Omega$ (depending on the scattering angle $\Omega$ ) is evaluated in the center of mass frame of the two colliding particles. Indices 3 and 4 label the momenta of the two particles after an elementary collision and we use the standard abbreviation $f_{i}=f\left(\mathbf{r}, \mathbf{p}_{i}, t\right)$. The collision is supposed elastic (conservation of energy, of total momentum). Pauli blocking factors $\left(1-f_{i} / 2\right)\left(1-f_{j} / 2\right)$ play an important role here, as they provide the necessary preservation of the Pauli principle in the course of fermion collisions. In the ground state, they block correctly all kinematically possible (and thus classically possible) collisions. At high excitation energy phase space opens up and two body collisions start to populate it in the course of thermalization. The VUU scheme was very much used in the case opf heavy-ion collisions in the Fermi energy domain. As we shall see below it should also be taken into account in the case of metal clusters, for energetic processes.

\section{FROM ONE FIELD TO THE NEXT}

\section{Status of knowledge}

The three fields (nuclei, clusters, helium droplets) are by no means at the same stage of developments. Nuclei are studied as such since almost a century while studies on free metal clusters have only been started a few decades ago, and even later for helium droplets, although the infinite phases of electrons (in bulk metal) or helium had been studied much earlier. As a result the available expertise varies from one field to the next. We have tried to summarize roughly the stages of achievement for each field in table 3, grouping into theory versus experiment and structure versus dynamics. Studies in nuclei cover each of the four topics widely. The case of clusters is more mixed. While structure properties start to be well known both at theoretical and experimental levels, dynamics is still in its infancy, especially 


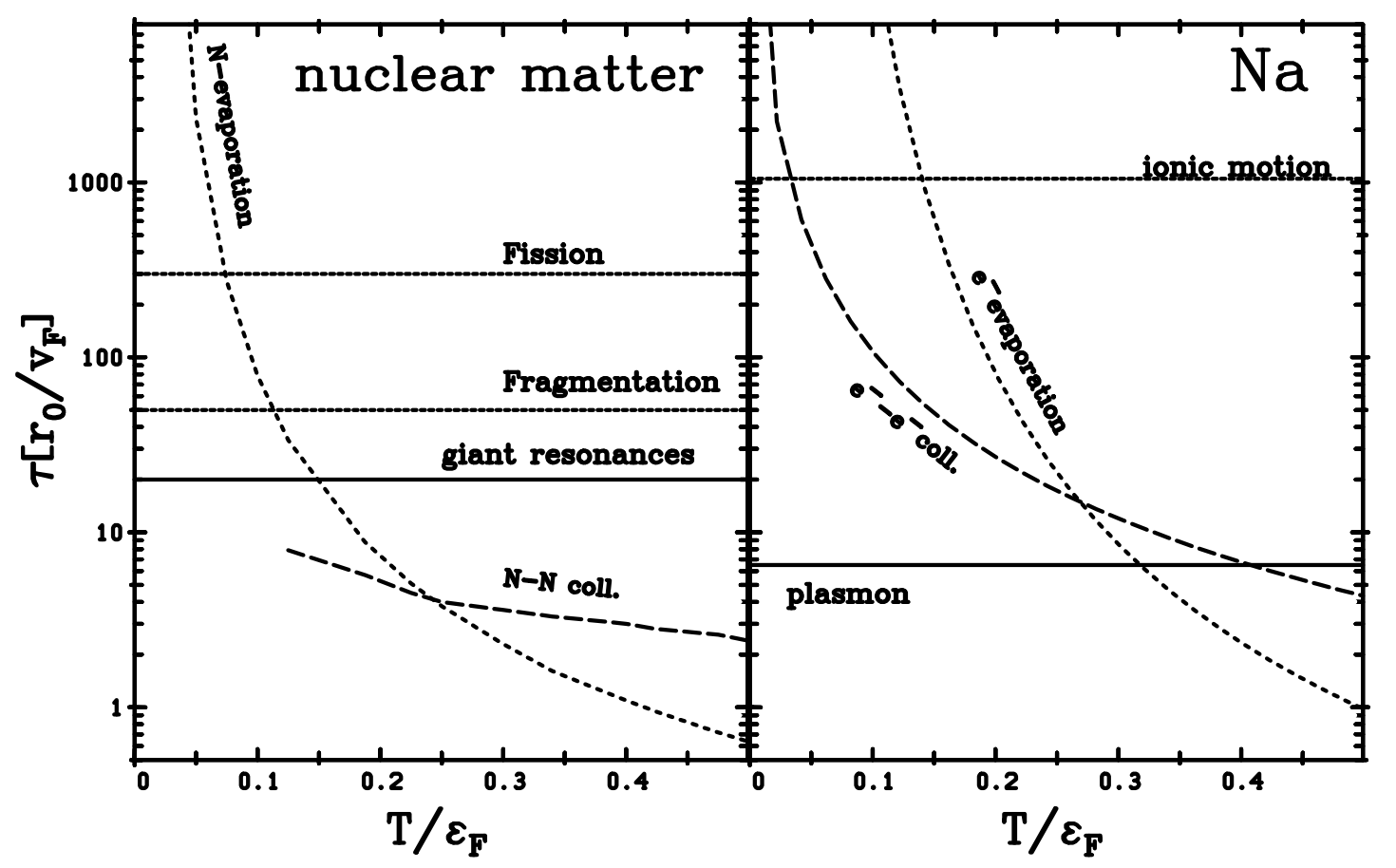

FIGURE 2. Comparison of relevant times scales in nuclei and sodium clusters. Reduced units are used in both cases to allow a relevant comparison (see text for details). Various times plotted are: plasmon period in clusters (equivalent to giant dipole resonance in nuclei), ionic time scale (comparable to nuclear fission/fragmentation), electron evaporation time (equivalent to neutron evaporation time), electron-electron (or nucleon-nucleon) collision time scale.

when far from equilibrium. Theoretical studies are here probably a bit more advanced than experimental ones. The latter require the development of very elaborate detectors with which the cluster community is not fully familiar and which would become unusually expensive in view of the else-wise rather economical cluster experiments. Data for helium droplets are even more sparse. In fact, there is very little known experimentally. Even the minimum size of such droplets is not fully ascertained experimentally. And it is admittedly very hard to deal with these volatile and neutral objects. The theory side stays a bit better at structure level. Dynamical studies are conceivable from the theory side, but there is not much effort in that direction because experimental data will not appear all to soon. In the context of this book focusing on nuclear dynamics, there are thus, presently, much stronger possible connexions between metal clusters and nuclei than with helium droplets. In the following we shall thus focus the discussion on dynamical examples as taken from metal clusters and not further analyze the case of helium droplets.

\section{Multiscale dynamics}

It is interesting, as a starter, to compare nuclear and cluster time scales. In order to make actual comparison more telling we use reduced units in terms of the Fermi gas characteristics of both systems, following the values introduced above. Indeed we define a basic time $r_{s, 0} / v_{F}$ and energy $\varepsilon_{F}$ scale, as built from the Wigner-Seitz radius $r_{s}$ for clusters and from the parameter $r_{0}$ of nuclear radius systematics $\left(R \sim r_{0} A^{1 / 3}\right.$, with $r_{0} \sim 1.12 \mathrm{fm}$ ). For sake of simplicity, we restrict the analysis of the cluster case to $\mathrm{Na}$, thus taking for the Wigner-Seitz radius $r_{s}=4 a_{0}$. This leads to $r_{s} / v_{F}=0.2$ fs and $\varepsilon_{F}=3.2 \mathrm{eV}$. Indeed, electronic time scales for other alkalines perfectly match the values obtained in the case of $\mathrm{Na}$. The ionic motion times scale with the square root of the atom mass. In nuclei the basic time and energy scales read $r_{0} / v_{F}=3.3 \mathrm{fm} / \mathrm{c}$ and $\varepsilon_{F}=40 \mathrm{MeV}$. We plot times as a function of temperature. It should be noted that this is rather a measure for the average excitation and does not necessarily imply a full thermalization. The choice of temperature is here practical and allows to overlook, to a large extent, size dependent effects.

With this system of reduced units we compare nuclear and sodium time scales in figure 2 . The comparison concerns various relevant times: the cluster plasmon period (equivalent to the giant dipole resonance in nuclei), ionic time scale 
(comparable to nuclear fission/fragmentation), electron (or neutron) evaporation time, electron-electron (or nucleonnucleon) time scale. This comparison calls for several comments. At first glance one can note a relative similarity between electronic and nuclear time scales, in particular comparable dependences (or independence) of times on temperature. But details differ. Indeed the hierarchy of time scales is pretty different between $\mathrm{Na}$ and nuclei. Grossly speaking, nuclear time scales look more similar to each other than cluster ones. This means that there exists a natural hierarchy of well separated time scales in clusters, while nuclear times tend to be much more mixed up. This has important implications in particular from the theoretical point of view. The lack of clear time hierarchy in nuclear dynamics makes a clean adiabatic decoupling of slow degrees of freedom difficult. The simple Born-Oppenheimer treatment of slow degrees of freedom has to be replaced by the much more involved generator-coordinate-method [16]. In cluster physics the huge mass difference between electron and ionic masses makes electron time scales an order of magnitude smaller than ionic ones. Electrons are thus more responsive than ions and need to be accounted for in priority in cluster dynamics. One should nevertheless note that the separation of electronic and ionic time scales tends to shrink in strongly non-linear situations where huge electromagnetic fields can be generated. Differences between nuclear and cluster hierarchies of time scales do not only reduce to the hierarchies by themselves but also to the times with respect to each other. One should in particular note the various importance of electron-electron interactions. They become dominant for much higher temperatures in clusters than in nuclei, which means that mean field methods can probably be used at much higher excitation energies in clusters than in nuclei. This is a welcome feature in view of the theoretical difficulties such effects raise. In a similar way, thermal emission comes into play much earlier in nuclei than in clusters. This again reflects the stronger interference amongst nuclear time scales as compared to cluster ones.

\section{ELECTRON DYNAMICS IN METAL CLUSTERS}

\section{Electron emission from irradiated clusters}

Experimental observation requires that some objects reach a counter, preferably charged particles. A major tool is here to keep a protocol of emitted electrons. Figure 3 illustrates the various observables which can be drawn from electron emission. The left upper panel symbolizes the photo-ionization cross section $\sigma(\omega)$ (where $\omega$ is the laser frequency) which is a good approximation to the total photo-absorption cross section and which can be measured easily by tracking the net electron yield as function of frequency. This quantity is obviously of inclusive nature and thus does not provide very detailed information, in particular at the side of individual electrons. More information can be extracted when measuring the distribution of the kinetic energies from the emitted electrons. This is called photoelectron spectroscopy (PES) and gives access to differential cross sections $d \sigma / d E$ (where $E$ is the electron kinetic energy). The right panels characterize PES briefly, in terms of mechanism (lower panel) and of typical observable (upper panel). The energy is drawn horizontally in both cases. The zero-point is clearly indicated. The lower part shows three vertical lines. The two solid lines indicate the (negative) energies of occupied bound electron states (in the simple case of a small cluster here) and the dashed line stands for the continuum threshold. The horizontal arrow indicates a photon. It transfers a well defined amount of energy. Bound state energy plus photon energy(ies) sum up to the kinetic energy finally observed. The photo-electron spectrum (upper panel) thus shows distinct peaks at those energies. Having these peaks and knowing the photon frequency, allows to conclude on the underlying single electron states. Thus far the simple story in the low intensity domain. The situation becomes more involved in a more energetic domain. The case of PES will be discussed in section .

\section{Photoelectron spectroscopy}

Figure 4 shows the PES of $\mathrm{Na}_{9}^{+}$for two different intensities around the transition to the high field regime. The lower intensity still resolves the detailed single-electron states in repeated sequences (see also the discussion around figure 6). A moderate enhancement of the laser intensity by an order of magnitude suffices to wipe out the structures. A more or less smooth curve then emerges which fits nicely to an exponential decrease. The smooth pattern persist, of course, for even larger intensities. The slope decreases with increasing intensity. It is interesting, then, to analyze the origin of these smooth pattern, a question which is still a matter of debate. Indeed one could interpret this exponential decrease as a signal of thermalization of the electron cloud. However, this is not applicable to short laser pulses during which thermalization can hardly play a dominant role. Without arguing in terms of thermalization one can also note 


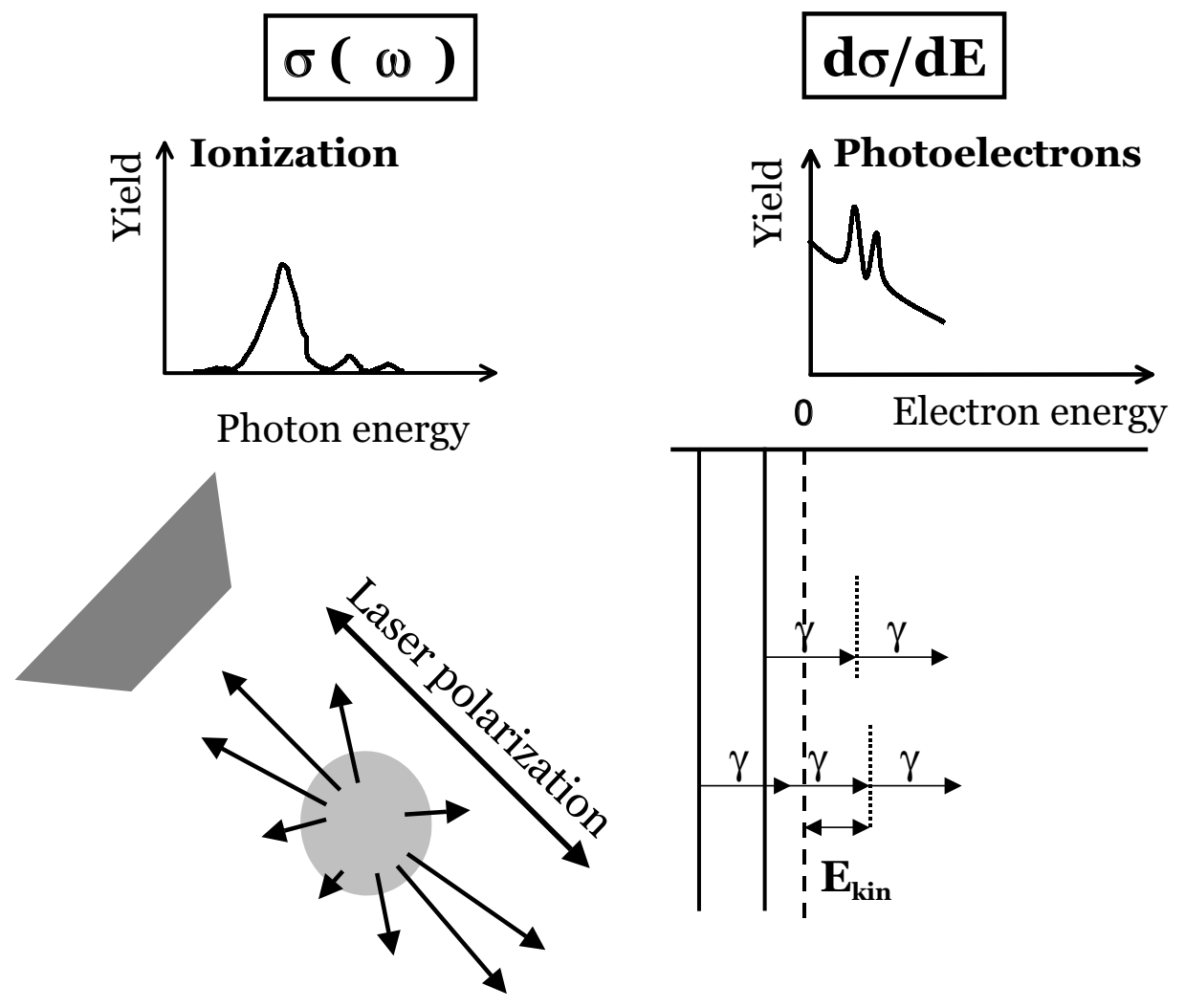

FIGURE 3. Schematic view of observables related to electron emission from metal clusters. Left lower panel: irradiated cluster with emission preferentially (to some extent) along laser polarization; a simple detector is also represented, its width symbolysing its capability to measure electron kinetic energies; Left upper panel: total ionzation cross section $\sigma(\omega)$ as a function of laser frequency $\omega$; Right panels: principle of photoelectron spectroscopy (upper panel: typical data, differential cross section $d \sigma / d E$ ); lower panel: mechanism).

that together with the dramatic changes in the pattern of the PES we see an equally dramatic increase in the ionization. This large increase in net charge at the cluster site has a side-effect on binding. Due to the growing Coulomb force, the mean field acquires extra binding, which globally down-shifts electronic single particle energies by the same amount. This happens as a dynamic process. Thus all levels are smeared which eventually generates the smooth pattern seen for the higher intensities. It can be shown that this process does also deliver an exponential decrease of the PES [17]. A better indicator of thermalization is provided by the (more detailed) analysis of electron emission in terms of the angular distribution of the emitted electrons. This will be discussed in section .

The PES change pattern when going to larger systems. Indeed, the larger the system, the denser the density of electronic states, which inhibits a detailed resolution of separate single electron states, whatever excitation regime. At best, one can expect step like structures indicating bands of occupied states, as was observed in the case of $\mathrm{C}_{60}$ for short, moderate pulses [18] and for large Ag clusters on substrate [19]. More recently were also published measurements on $\mathrm{Na}_{93}^{+}$from [20], which show smooth trends throughout and are interpreted as thermal emission. Let us thus consider here $\mathrm{Na}_{93}^{+}$as an example of a larger cluster. We have computed PES for $\mathrm{Na}_{93}^{+}$for a variety of laser intensities (but fixed photon frequency and pulse width). At low intensity we observe step like pattern related to a dense block of occupied states. And at larger intensity PES exhibit smooth pattern with nearly exponential decrease. A simple characteristics of the PES is thus provided by the slope of the exponential decrease. The criterion is unambiguous at large intensity but requires some caution at lower intensities because of the step like pattern. The "'staircases"' have in fact all the same step height (on 'logarithmic scale) such that their envelope is a straight line to which it easy to associate an exponential decrease. We can thus extend the simple slope characterization to any laser intensity. This allows a direct comparison to experimental data. 


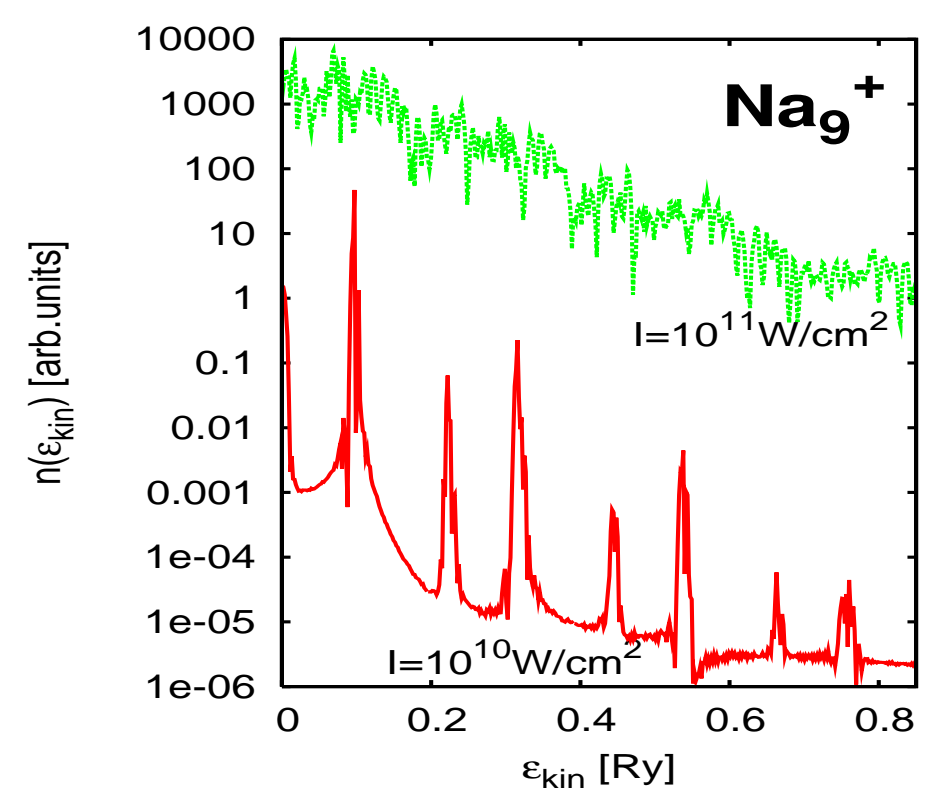

FIGURE 4. PES of $\mathrm{Na}_{9}^{+}$at two intensities as indicated. A short laser pulse with FWHM $=25 \mathrm{fs}$ was used.

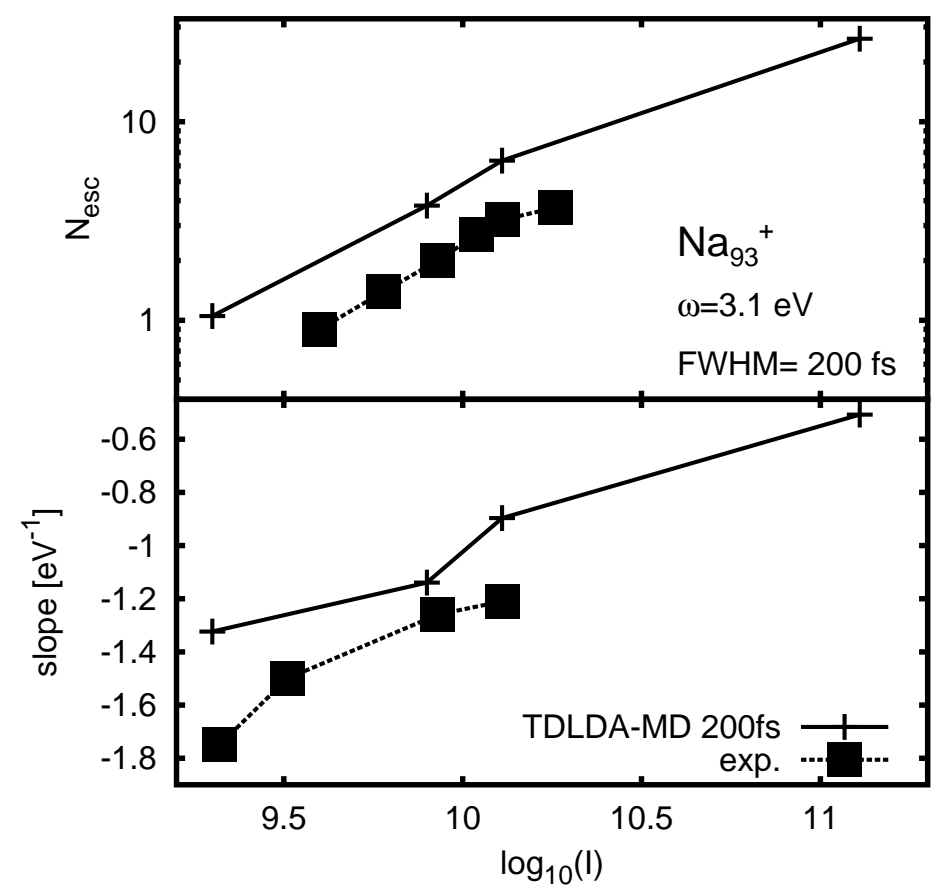

FIGURE 5. Global properties of emission from $\mathrm{Na}_{93}^{+}$, total number of emitted electrons (gain in ionization) in the upper panel and slope of the PES in the lower panel. The laser parameters were $\omega_{\text {photon }}=3.1 \mathrm{eV}$ and pulse length FWHM=200 fs. Results are drawn versus intensity $\left(\right.$ as $\log _{10}(I)$ with $I$ in units of $\mathrm{W} / \mathrm{cm}^{2}$ ). Results from TDLDA-MD are compared with the experimental results of [20] using comparable experimental conditions. 
The experiments in [20] were done for rather long pulses with a FWHM of about $200 \mathrm{fs}$. One can thus expect to see pattern related to electron electron collisions, beyond mean-field. Nonetheless, it is interesting to compare TDLDA (mean-field) results with those measurement concerning global properties as net ionization and the slope of the PES. The results are compared with data in figure 5. Note that for such long pulses one has also to account for ionic motion which sizably alter the sequence of electronic levels and thus the PES. This effect was of course taken into account in the calculations presented in figure 5. The comparison shows that the calculations reproduce the net ionization (number of emitted electrons $N_{\text {esc }}$ within a factor of two, as well as the growth with laser intensity is perfectly reproduced. This has to be considered as a good agreement in view of the fact that ionization also sensitively depends on the pulse shape. Calculations use here a $\cos ^{2}$ while the experimental profile is not so well known, probably having longer tails. The results for the slopes (lower part of figure 5) are also quite encouraging in size and in trend. Similar results were reported in [21] in the framework of a Vlasov-LDA approach. The remaining differences between the calculations and the experimental results are presumably to be attributed to the lack of account of electron electron collisions (overlooking details of laser pulse shape, as mentioned above or even cluster temperature control).

\section{Angular distributions}

Besides the kinetic energy, one can also measure the angular distribution of emitted electrons, a quantity which also carries a lot of interesting information. This gives access to the differential cross section $d \sigma / d \Omega$ (where $\Omega$ is the solid angle).The principle of such a measure is presented schematically in figure 6 , which is so to say an update of figure 3. In the case of laser irradiation one expects electrons to be emitted preferentially, at least to some extent, along laser polarization axis. As we shall see below the amount of anisotropy somewhat depends on the experimental conditions (characteristics of laser pulse in particular). One may even measure simultaneously both angular distributions and kinetic energies of emitted electrons (see left panel figure 7 for an example). This thus gives access to the double differential cross section $d^{2} \sigma / d \Omega d E$. Note also that in figure 6 the PES panel (upper right part) has been modified, with respect to figure 3 , in order to describe the multiphoton regime, with successive copies of the single electron level sequence.

There are only few available experimental data on angular distributions. An example is shown in figure 7. The left panel exhibits a combined kinetic energy and angular distribution measurement, after irradiation of a $\mathrm{W}_{4}{ }^{-}$cluster anion by a low-intensity ns laser at a frequency of $4 \mathrm{eV}$. The anion (negatively charged cluster) has a low ionization threshold around $1.6 \mathrm{eV}$, much lower than the monomer evaporation threshold (larger than $7 \mathrm{eV}$ ). As a result, thermal ionization is favored over monomer evaporation in this case. The competition remains, though, between direct (in particular one-photon processes in such an anion) and thermal electron emission. The extremely long laser pulse (as compared to typical electronic or even ionic times) gives thermalization through electron-electron collisions good chances to be activated and efficient. One thus expects a significant contribution from thermal emission. This is indeed what can be seen from the figure, where light grey indicates large emission and dark grey low emission. The broad central spot can be associated with thermal (isotropic) emission and the kinetic energy spectra (not directly visible in the figure) indeed confirm the correct trend $\propto \sqrt{\varepsilon_{\text {kin }}} \exp \left(-\varepsilon_{\text {kin }} / T\right)$ [22]. But at larger kinetic energies (which correspond in this representation to larger radial distances) one can also spot a non-isotropic component in the emission, directed along the laser polarization axis. This is clearly a signal from a direct emission process which competes with thermal (isotropic) emission.

A full description of such processes should thus account for electron-electron collisions in order to properly access the isotropic component of the electronic emission. This would allow to cover both regimes, direct emission as well as thermal evaporation. A way to include such effects in the TDLDA approach is to rely on the semi classical version of the theory, properly extended by a collision term to account for electron electron collisions [23, 24], in the spirit of similar extensions worked out several years ago in nuclear physics [15]. The semi-classical approach of course requires sufficiently high excitation. But, as in the nuclear context, that is the typical situation for thermalization to play a role at all. The right panel of figure 7 shows an example of angular distributions obtained from such a VUU calculation, and compared to pure mean-field results (a quantum TDLDA one and a semi classical Vlasov-LDA one). Note that at variance with the left panel of figure 7 the distribution has been integrated over final kinetic energy. First, we see that TDLDA and Vlasov nicely agree in that excitation regime. Both show an emission clearly peaked along the laser polarization, a behavior characteristic of direct emission. But the electron-electron collisions in VUU also leads to a sizeable isotropic component. Not surprisingly, the delayed emission of the thermalized electrons has lost memory of the original polarization axis and subsequently one obtains a much smoother angular distribution, as can 


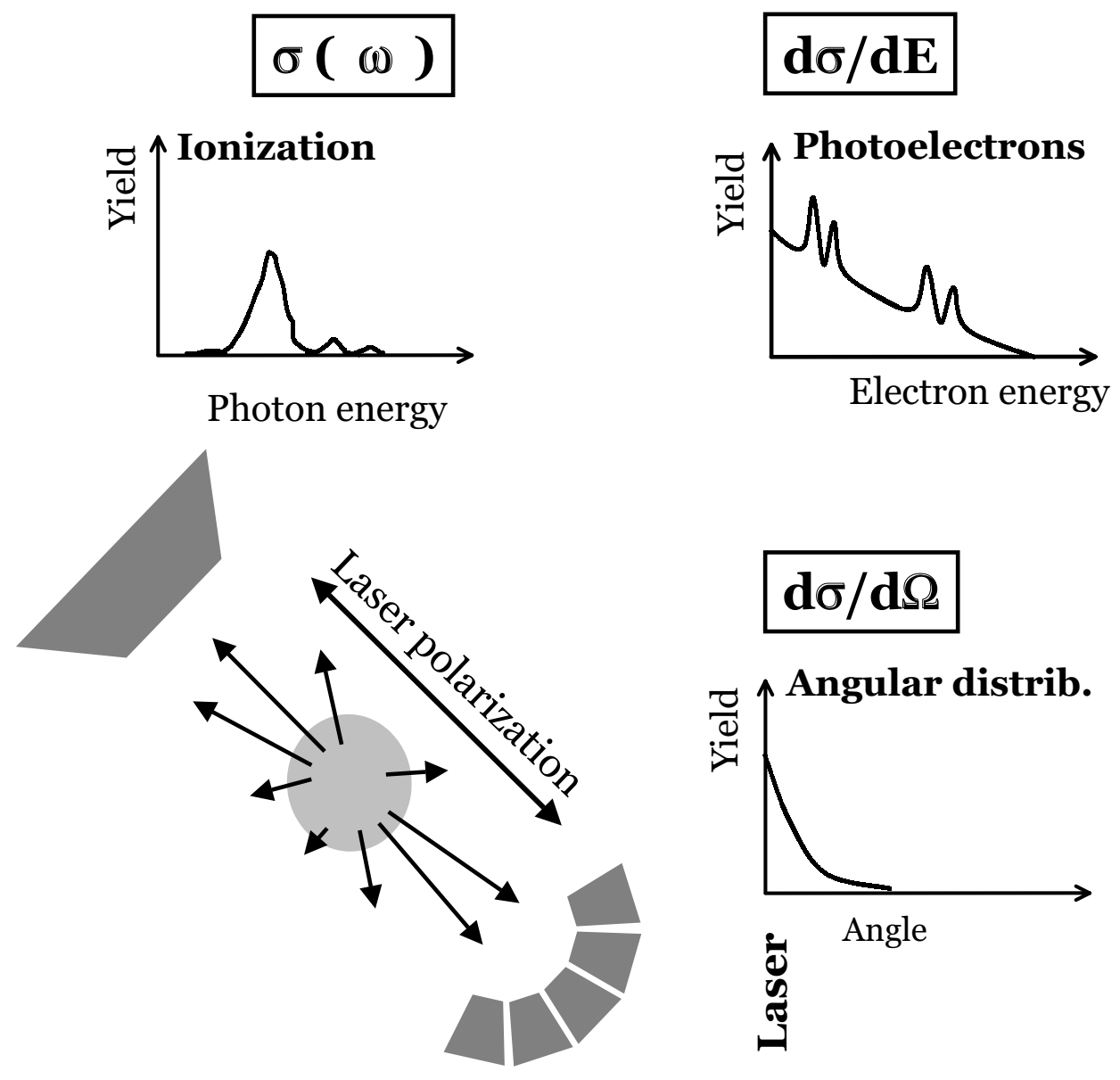

FIGURE 6. Schematic view of detailed characteristics of emitted electrons, in particular in the non linear domain. The left panels of the figure are similar to the ones of figure 3 but for the detectors (left lower panel) where we have schematized a series of small detectors to access angular distributions of emitted electrons (and possibly even kinetic energies at the same time). Right upper panel: PES in the multiphoton regime with copies of the series of single particle peaks separated by the laser frequency, yielding the differential cross section $d \sigma / d E$; Right lower panel: angular distribution of emitted electrons with a more intense yield along laser polarization axis, yielding the differential cross section $d \sigma / d \Omega$ and possibly the double differential cross section $d^{2} \sigma / d \Omega d E$.

be seen on the VUU curve of the right panel of figure 7. The distribution is nevertheless not perfectly isotropic: there remains a sizeable fraction of directly emitted electrons for the chosen conditions. But the branching between direct and thermal emission in fact sensitively depends on the details of the excitation. Systematic studies of these influences could thus deliver valuable information on the underlying dynamics. But these studies have yet to be worked out, both theoretically and experimentally, in particular in the combined analysis of kinetic energies and angular distribution as in the case presented in the left panel of figure 7.

\section{SOME CONCLUSIONS AND PERSPECTIVES}

Fermi liquids are a generic state of matter denoted by a more or less well defined saturation point and a long mean free path for particles with low momenta. Wigner-Seitz radius (related to saturation density) and Fermi energy set natural units for length and energy scales. Finite drops of Fermi liquid show several interesting features as, e.g., pronounced shell effects and resonance excitations. Various different materials look very similar when expressed in the natural units of the material. We have exemplified that here for the three systems: nuclei, metal clusters and ${ }^{3} \mathrm{He}$ droplets. There are, of course, also many differences between these systems. These concern mostly availability and experimental 

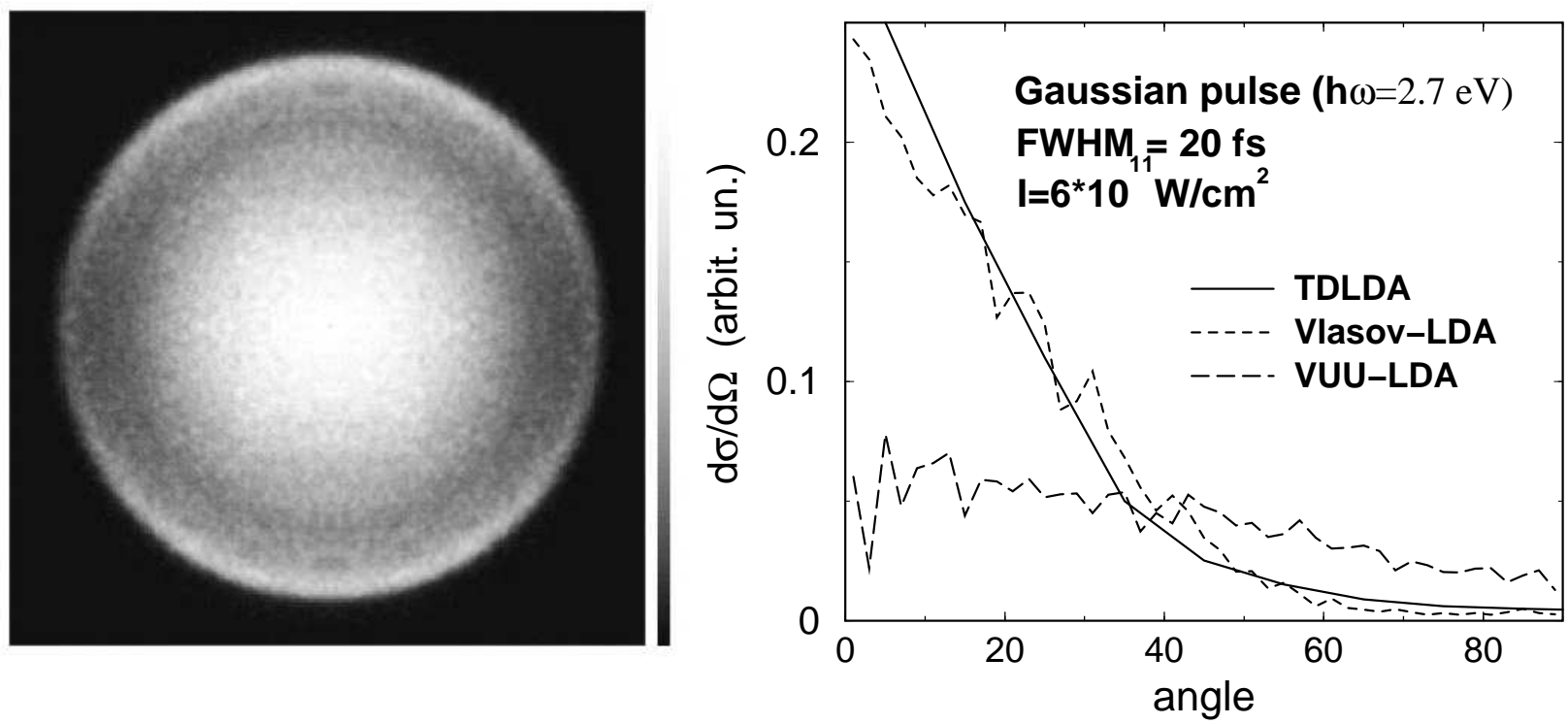

FIGURE 7. Left panel: $2 \mathrm{D}$ equi-density plot of kinetic energy spectra and angular distribution from a $W_{4}^{-}$cluster anion after irradiation with a laser of frequency $4 \mathrm{eV}$. The emission angle is mapped in terms of polar coordinates while the kinetic energies grow with radial distance from the center of the plot. The gray scale indicates flux: high emission shines white. The laser polarization is along the vertical axis [22]. Right panel: angular distribution of emitted electrons computed in quantum TDLDA (dotted), semiclassical Vlasov-LDA (dashed), and VUU with collision term (full line). Test case is $\mathrm{Na}_{41}^{+}$with ionic structure. Laser parameters are indicated in the figures. The angle is defined relative to the laser polarization axis.

access. For example, nuclei are limited in size, but metal clusters can be grown arbitrarily large which allows to study the approach to bulk matter and the evolution of shell effects for large systems. Furthermore, clusters are responsive to laser light which opens up an enormously rich field of dynamical studies yet to come. As another example, ${ }^{3} \mathrm{He}$ droplets have extremely soft surfaces which requires an extension of DFT to incorporate effective interactions with finite range. This shows that the differences are highly welcome as they deliver complementing information for a common understanding of finite Fermion systems. It is to be remarked that nuclei are a particularly demanding species in several respects. The nuclear many-body problem (ab initio models) has not yet fully converged and similarly the nuclear DFT, although extremely successful in sorting the basic nuclear properties, is still under development. On the other hand, nuclei are probably the best studied objects from the experimental side. There exist plentiful of data in any dynamical regime. Altogether, a combined analysis of the different systems will mutually boost the understanding of each one of the systems.

Pursuing the comparative analysis a bit deeper it seems clear, in particular in relation with the examples shown above, that there obviously exist rather clear directions of fruitful enrichment, expecially in the domain of dynamics. Indeed, nuclear physics, and heavy ion collisions, provide remarkable examples of detailed studies of complex dynamical processes requiring sophisticated multi parameter detectors and elaborate many body theories beyond the mere effective mean-field. It seems to us that the field of metal clusters should easily benefit from this knowhow, as it has already benefited in the case of low energy dynamics or in some structure properties. We have outlines, in particular, the importance of accessing as detailed as possible information on emitted electrons from irradiated clusters. And its seems that all the experience gathered around multidetectors in heavy ion collisions could here be very useful. The essential step, to pass from inclusive to exclusive measurements has been extensively explored in these nuclear studies and the experience gained here appears quite valuable for other domains of physics.

This is however not the single direction to be explored. Fundamental cross disciplinary questions also arise in other areas at the interface between nuclear and cluster physics. Let us in particular cite the question of "phase transitions" in finite systems, a problem extensively studied in relation to nuclear fragmentation and to melting in metal clusters. This particular question is addressed elsewhere in this book. We have thus deliberately avoided this point. From a more formal point of view, one should also mention the basic questions raised by Density Functional Theory in various systems (electronic, nuclear as well as helium). The point is especially true in relation, again, to dynamical questions. 
The development of the so-called Time Dependent Density Functional Theory (TDDFT) still remains a matter of intense activity and debates. The nuclear physics approach, for example in terms of truncations of hierarchies of density matrices, brings here an interesting light, to be merged with the more "bottom-up" methods inherited from standard DFT methods. Finally we would like again to mention, in continuity to these questions on DFT and TDDFT, the growing importance of dynamical correlations in more and more energetic processes. Again the nuclear physics experience, for example in terms of kinetic theory, provides valuable assets for other fields of physics and this should be valorized.

Acknowledgements. The authors thank the french german exchange programm PROCOPE, the CNRS program "Matériaux", the Institut Universitaire de France, the Humboldt Foundation and the french ministry of research (Gay Lussac) for financial support during the realization of this work.

We list in the following a few textbooks used in the preparation of this manuscript, as well the proceedings of a few major conferences on cluster and helium physics. This list is by no means exhaustive.

\section{- Cluster Physics}

- S Sugano,Microclusters Springer, Berlin (1987)

- H Haberland, Clusters of Atoms and Molecules 1 - Theory, Experiment, and Clusters of Atoms, Springer Series in Chemical Physics, vol. 52, Berlin (1994)

- H Haberland, Clusters of Atoms and Molecules 2 - Solvation and Chemistry of Free Clusters, and Embedded, Supported and Compressed Clusters, Springer Series in Chemical Physics, vol. 56, Berlin (1994)

- W Ekardt,Metal Clusters, Wiley, New York (1999)

- J Jellinek,Theory of Atomic and Molecular Clusters, Springer, Berlin (1999)

- P G Reinhard and E Suraud,Introduction to cluster dynamics, Wiley-VCH, Berlin (2003)

- Proceedings of the ISSPIC conferences of the past decade:,

ISSPIC 7: Surf. Rev. Lett. 3 (1996)

ISSPIC 8: Z. Phys. D 40 (1997)

ISSPIC 9: Eur. Phys. J. D 9 (1999)

ISSPIC 10: Eur. Phys. J. D 16 (2001)

ISSPIC 11: Eur. Phys. J. D 24 (2003)

ISSPIC 12: Eur. Phys. J. D 2 (2005)

\section{- Helium clusters}

- E R. Dobbs, Helium Three, Oxford University Press, New York, (2000)

- K B Whaley (editor), Special issue of The Journal of Chemical Physics, 115, (2001)

- E. Krotscheck and J Navarro (Eds.), Microscopic Approaches to Quantum Liquids in Confined Geometries, World Scientific, Singapore (2002)

\section{REFERENCES}

1. D Pines and P Nozières. The Theory of Quantum Liquids. W A Benjamin, New York, 1966.

2. M Brack. Rev. Mod. Phys., 65:677, 1993.

3. G F Bertsch and R A Broglia. Oscillations in Finite Quantum Systems. Cambridge University Press, Cambridge, 1994.

4. U Näher, S Björnholm, S Frauendorf, F Garcias, and C Guet. Phys. Rep., 285:245, 1997.

5. R M Dreizler and E K U Gross. Density Functional Theory: An Approach to the Quantum Many-Body Problem. Springer-Verlag, Berlin, 1990.

6. M. Bender, P.-H. Heenen, and P.-G. Reinhard. Self-consistent mean-field models for nuclear structure. Rev. Mod. Phys., 75:121, 2003.

7. S. Weisgerber and P.-G. Reinhard. The shell structure of ${ }^{3}$ He droplets. Z. Phys. D, 23:275, 1992.

8. P-G Reinhard and E Suraud. page 211. Wiley, New York, 1999.

9. P-G Reinhard and E Suraud. Introduction to Cluster Dynamics. Wiley, New York, 2003.

10. P. Ring and P. Schuck. The Nuclear Many-Body Problem. Springer-Verl., New York, Heidelberg, Berlin, 1980.

11. M. Barranco, J. Navarro, and A. Poves. Phys Rev. Lett., 78:4729, 1997.

12. S. Weisgerber and P.-G. Reinhard. Phys. Rev. Lett., 84:3121, 2000. 
13. E.R. Dobbs. Helium Three. Oxford University Press, New York,, 2000.

14. R. Guardiola and J. Navarro. Phys Rev. Lett., 84:1144, 2000.

15. G F Bertsch and S Das Gupta. Phys. Rep., 160:190, 1988.

16. P.-G. Reinhard and K. Goeke. The generator coordinate method and quantised collective motion in nuclear systems. Rep. Prog. Phys., 50:1, 1987.

17. A. Pohl, P.-G. Reinhard, and E. Suraud. Exponential photoelectron spectra in na clusters. J. Phys. B, 37:3301, 2004.

18. E E B Campbell, K Hansen, K Hoffmann, G Korn, M Tchaplyguine, M Wittmann, and I V Hertel. Phys. Rev. Lett., 84:2128, 2000.

19. J Lehmann, M Merschdorf, W Pfeiffer, A Thon, S Voll, and G Gerber. Phys. Rev. Lett., 85:2921, 2000.

20. R Schlipper, R Kusche, B von Issendorff, and H Haberland. Appl. Phys. A, 72:255, 2001.

21. T Fennel, G F Bertsch, and K-H Meiwes-Broer. Euro. Phys. J. D, 29:367, 2004.

22. B Baguenard, J C Pinar, C Bordas, and M Broyer. Phys. Rev. A, 63:023204, 2001.

23. A Domps, P-G Reinhard, and E Suraud. Phys. Rev. Lett., 80:5520, 1998.

24. E Giglio, P-G Reinhard, and E Suraud. J. Phys. B, 34:1253, 2001. 